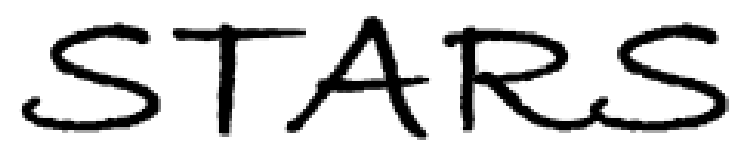

University of Central Florida

STARS

$1-1-2011$

\title{
Multimode fiber amplifier with tunable modal gain using a reconfigurable multimode pump
}

\author{
Neng Bai \\ University of Central Florida \\ Ezra Ip \\ Ting Wang \\ Guifang Li \\ University of Central Florida
}

Find similar works at: https://stars.library.ucf.edu/facultybib2010

University of Central Florida Libraries http://library.ucf.edu

This Article is brought to you for free and open access by the Faculty Bibliography at STARS. It has been accepted for inclusion in Faculty Bibliography 2010 s by an authorized administrator of STARS. For more information, please contactSTARS@ucf.edu.

\section{Recommended Citation}

Bai, Neng; Ip, Ezra; Wang, Ting; and Li, Guifang, "Multimode fiber amplifier with tunable modal gain using a reconfigurable multimode pump" (2011). Faculty Bibliography 2010s. 7063.

https://stars.library.ucf.edu/facultybib2010/7063

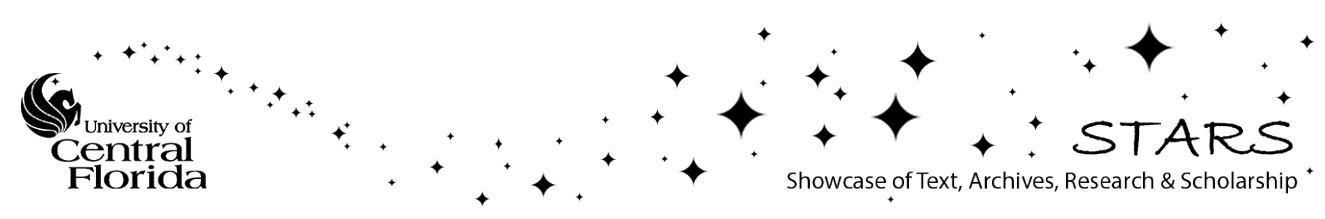




\title{
Multimode fiber amplifier with tunable modal gain using a reconfigurable multimode pump
}

\author{
Neng Bai, ${ }^{1,2,}$ Ezra Ip, ${ }^{2}$ Ting Wang, ${ }^{2}$ and Guifang $\mathrm{Li}^{1}$ \\ ${ }^{1}$ College of Optics \& Photonics/CREOL\&FPCE, University of Central Florida, 4000 Central Florida Boulevard, \\ Orlando, Florida 32816-2700, USA \\ ${ }^{2}$ NEC Laboratories America, Inc. 4 Independence Way, Suite 200, Princeton, New Jersey 08540, USA \\ *bneng@creol.ucf.edu
}

\begin{abstract}
We propose a method for controlling modal gain in a multimode Erbium-doped fiber amplifier (MM-EDFA) by tuning the mode content of a multimode pump. By adjusting the powers and orientation of input pump modes, modal dependent gain can be tuned over a large dynamic range. Performance impacts due to excitation of undesired pump modes, mode coupling and macro-bending loss within the erbium-doped fiber are also investigated. The MM-EDFA may potentially be a key element for long haul mode-division multiplexed transmission.
\end{abstract}

(C2011 Optical Society of America

OCIS codes: (060.2360) Fiber optics amplifiers and oscillators; (060.2330) Fiber optics communications.

\section{References and links}

1. D. Qian, M.-F. Huang, E. Ip, Y.-K. Huang, Y. Shao, J. Hu, and T. Wang, “101-Tb/s (370×294-Gb/s) PDM128QAM-OFDM transmission over $3 \times 55$-km SSMF using pilot-based phase noise mitigation," in Proc. OFC (Los Angeles, CA, USA 2011). Paper PDPB5.

2. H. T. Hattori and A. Safaai-Jazi, "Fiber designs with significantly reduced nonlinearity for very long distance transmission," Appl. Opt. 37(15), 3190-3197 (1998).

3. F. Yaman, N. Bai, Y.-K. Huang, M.-F. Huang, B. Zhu, T. Wang, and G. Li, "10 x 112Gb/s PDM-QPSK transmission over $5032 \mathrm{~km}$ in few-mode fibers," Opt. Express 18(20), 21342-21349 (2010).

4. G. J. Foschini, "Layered space-time architecture for wireless communications in a fading environment when using multielement antennas," Bell Labs Tech. J. 1(2), 41-59 (1996).

5. J. Sakaguchi, Y. Awaji, N. Wada, A. Kanno, T. Kawanishi, T. Hayashi, T. Taru, T. Kobayashi, and M. Watanabe, "109-Tb/s (7×97×172-Gb/s SDM/WDM/PDM) QPSK transmission through 16.8-km homogeneous multi-core fiber," Proc. OFC 2011, Paper PDPB6, Los Angeles, CA, USA (2011).

6. B. Zhu, T. G. Taunay, M. Fishteyn, X. Liu, S. Chandrasekhar, M. F. Yan, J. M. Fini, E. M. Monberg, F. V. Dimarcello, K. Abedin, P. W. Wisk, D. W. Peckham, and P. Dziedzic, "Space-, wavelength-, polarizationdivision multiplexed transmission of 56-Tb/s over a 76.8-km seven-core fiber," Proc. OFC 2011, Paper PDPB7, Los Angeles, CA, USA (2011).

7. A. Li, A. A. Amin, X. Chen, and W. Shieh, "Reception of mode and polarization multiplexed 107-Gb/s COOFDM signal over a two-mode fiber," Proc. OFC 2011, Paper PDPB8, Los Angeles, CA, USA (2011).

8. M. Salsi, C. Koebele, D. Sperti, P. Tran, P. Brindel, H. Mardoyan, S. Bigo, A. Boutin, F. Verluise, P. Sillard, M. Astruc, L. Provost, F. Cerou, and G. Charlet, "Transmission at $2 \times 100-\mathrm{Gb} / \mathrm{s}$ over two modes of $40 \mathrm{~km}-\mathrm{long}$ prototype few-mode fiber, using LCOS based mode multiplexer and demultiplexer," Proc. OFC 2011, Paper PDPB9, Los Angeles, CA, USA (2011).

9. R. Ryf, S. Randel, A. H. Gnauck, C. Bolle, R.-J. Essiambre, and P. J. Winzer, "Space-division multiplexing over $10 \mathrm{~km}$ of three-mode fiber using coherent $6 \times 6$ MIMO processing," Proc. OFC 2011, Paper PDPB10, Los Angeles, CA, USA (2011).

10. P. M. Krummrich and K. Petermann, "Evaluation of potential optical amplifier concepts for coherent mode multiplexing," Proc. OFC 2011, Paper OMH5, Los Angeles, CA, USA (2011).

11. E. Desurvire, Erbium-doped Fiber Amplifiers-Principles and Applications, (John Wiley \& Son Inc. 1994), Chap. 1.

12. C. D. Stacey and J. M. Jenkins, "Demonstration of fundamental mode propagation in highly multimode fibre for high power EDFAs," Conference on Lasers and Electro-Optics Europe (CLEO 2005), Munich, Germany, June 17 , p. 558.

13. A. Galvanauskas, "Mode-scalable fiber-based chirped pulse amplification systems," IEEE J. Sel. Top. Quantum Electron. 7(4), 504-517 (2001).

14. M. Gong, Y. Yuan, C. Li, P. Yan, H. Zhang, and S. Liao, "Numerical modeling of transverse mode competition in strongly pumped multimode fiber lasers and amplifiers," Opt. Express 15(6), 3236-3246 (2007).

\#147622 - \$15.00 USD Received 16 May 2011; revised 27 Jun 2011; accepted 7 Jul 2011; published 15 Aug 2011

(C) 2011 OSA

15 August 2011 / Vol. 19, No. 17 / OPTICS EXPRESS 16601 
15. D. Gloge, "Weakly guiding fibers," Appl. Opt. 10(10), 2252-2258 (1971).

16. C. D. Poole and S.-C. Wang, "Bend-induced loss for the higher-order spatial mode in a dual-mode fiber," Opt. Lett. 18(20), 1712-1714 (1993).

17. D. Marcuse, "Curvature loss formula for optical fibers," J. Opt. Soc. Am. 66(3), 216-220 (1976).

\section{Introduction}

Over the past years, advances in optical coherent detection and signal processing have led to tremendous growth in the spectral efficiency achieved in optical fiber. Recently, 100-Tb/s transmission at a spectral efficiency of $11 \mathrm{~b} / \mathrm{s} / \mathrm{Hz}$ was reported in a single-mode fiber [1]. Owing to the nonlinear refractive index of silica, it is impossible to continue increasing spectral efficiency indefinitely by merely increasing the launched power. One method to reduce fiber nonlinearity is to increase the effective area of the propagating mode, thus reducing the optical intensity and the resulting nonlinear effects [2]. However, mode effective area is limited by bending loss and by the requirement of the waveguide to be single-mode. It is possible to transmit data in the fundamental mode of a "few-mode fiber" (FMF). Provided mode coupling is low, the signal will remain single-mode during propagation. The larger effective area of the fundamental mode in FMF in comparison with that achievable in SMF can further reduce nonlinearity [3]. However, a nonlinear capacity limit will always exist. Even if the transmission medium was linear, Shannon's capacity $C=B_{W} \log _{2}(1+S N R) \mathrm{b} / \mathrm{s}$ per channel shows that the capacity scales only logarithmically with signal-to-noise ratio. Ultra-high spectral efficiency is therefore very power inefficient. To achieve cost-effective scaling in system capacity, new paradigms in optical transmission are required.

One promising solution is space-division multiplexing, where data is transmitted over parallel channels. Indeed, transmission over parallel orthogonal channels has been well established in wireless systems, where the achievable capacity using multiple-input multipleoutput (MIMO) antennas increases with the number of independent "eigenchannels," which under the assumption of rich multipath, scales as the minimum of the number of antennae deployed at the transmitter and receiver [4].

In optical fiber transmission, two space-division multiplexing (SDM) schemes have been proposed. These are (i) multicore fibers $(\mathrm{MCF})$, where a single strand of glass fiber contains a number of independent single- (or multi-) mode cores each capable of communicating optical signals [5,6]; and (ii) multimode fibers (MMF), where a single strand of fiber has one core with sufficiently large cross-section area to support a number of independent guiding modes [7-9]. SDM transmission experiments have been reported for both types of fibers. Owing to the lack of available inline amplifiers, all MCF and MMF experiments to date have been single-span, with transmission distances up to $76.8 \mathrm{~km}$ [6] for MCF and 40km [8] for MMF.

To enable mode-division multiplexing (MDM) in MMF over long-haul distances, inline erbium-doped fiber amplifiers (EDFA) based on MMF are required [10]. The theory of multimode EDFAs (MM-EDFA) has been studied in [11]. Applications for MM-EDFAs have included high-powered lasers and free-space communications, where the multimode optical waveguide is essentially used in a "single-mode" manner, thus mode-dependent gain (MDG) is not critical [11,12]. In MDM transmission however, careful control over MDG is necessary to overcome mode-dependent loss (MDL) in the transmission fiber, and to ensure all signal modes are launched with optimal power maximizing the total system capacity.

Mode dependent gain (MDG) is mainly determined by three factors: (a) the concentration profile of the active dopant ions, (b) the transverse intensity profile of the pump, and (c) the transverse intensity profile of the signal. In general, a signal mode whose profile is better matched to the pump intensity profile will experience higher gain. Hence, by controlling the mode content of the pump, it is possible to control MDG. The organization of this paper is as follows. In Section 2, we review the theory of MM-EDFAs. In Section 3, we provide simulation results for a step-index "two-mode fiber," demonstrating the feasibility of MDG 
control by tuning the mode content of the pump. We also explore the dependence of MDG on the excitation of unwanted modes and mode coupling within the EDF.

\section{Theory}

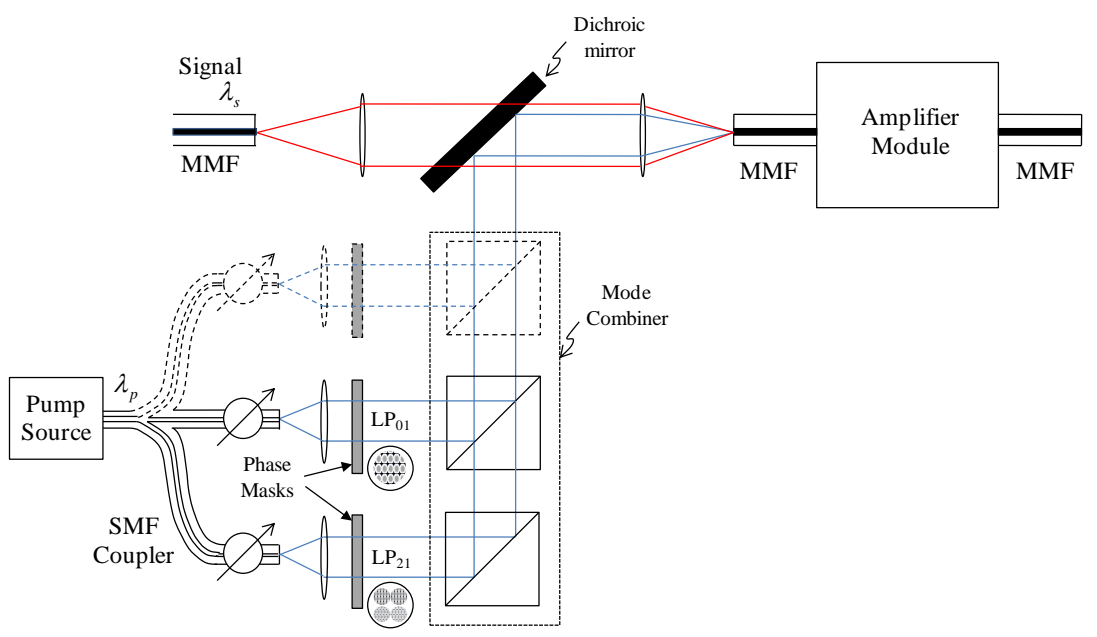

Fig. 1. Schematic diagram of an MM-EDFA.

A schematic of a MM-EDFA is shown in Fig. 1. To generate the desired pump intensity profile, we split the pump source into $N$ paths, and use mode converters to transform the spatial mode of the pump source into the $N$ spatial modes of the MMF. The variable attenuators enable $N$-degree control over the mode content of the pump, and thus the MDG of the device. The pump modes are spatially combined with the signal, which are injected into the erbium-doped MMF. In the paper, we assume the erbium-doped MMF has the profile shown in Fig. 2, where the core has radius $r_{c}$, and a region of the core for which $r<a \leq r_{c}$ is doped with Erbium atoms at a concentration of $N_{0}(r, \varphi)$.

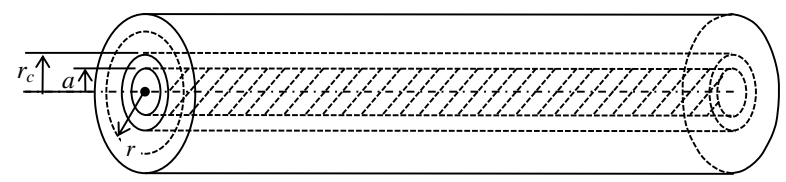

Fig. 2. Multimode Erbium-doped fiber amplifier.

The operation of a multimode fiber amplifier is described by coupled differential equations involving: (i) evolution of the intensities of the various signal and pump modes along the amplifying medium, and (ii) population inversion along the amplifying medium [11]. In contrast to a single-mode EDFA, the transverse intensity distributions have to be taken into account in a multimode EDFA. We assume both signal and pump are copropagating. Let $\Gamma_{s, i}(r, \varphi)$ and $\Gamma_{p, j}(r, \varphi)$ be the normalized intensity patterns of the $i$-th signal mode and $j$-th pump mode of the EDF, respectively; and let $P_{s, i}$ and $P_{p, j}$ be their respective power. We further assume the erbium-doped fiber (EDF) can be modeled as a quasi-three-level system at $980 \mathrm{~nm}$ pumping, and let $N_{1}(r, \varphi, z)$ and $N_{2}(r, \varphi, z)$ with $N_{1}(r, \varphi, z)+N_{2}(r, \varphi, z)=N_{0}(r, \varphi)$ be the population densities of Erbium atoms in the lower and upper levels at position $(r, \varphi, z)$. Loss is assumed negligible in the EDF. It can be shown 
that the intensity evolution equations for signal and amplified spontaneous emission (ASE) in the i-th signal mode at the wavelength $\lambda_{s}$ are given by:

$$
\begin{aligned}
& \frac{d P_{s, i}}{d z}=P_{s, i} \int_{0}^{2 \pi} \int_{0}^{a} r d r d \varphi \Gamma_{s, i}(r, \varphi)\left[N_{2}(r, \varphi, z) \sigma_{e s, i}-N_{1}(r, \varphi, z) \sigma_{a s, i}\right]-\sum_{k=1}^{m_{s}} d_{s, i \leftrightarrow k}\left[P_{s, i}-P_{s, k}\right] \\
& \frac{d P_{A S E, i}}{d z}= P_{A S E, i} \int_{0}^{2 \pi} \int_{0}^{a} r d r d \varphi \Gamma_{s, i}(r, \varphi)\left[N_{2}(r, \varphi, z) \sigma_{e s, i}-N_{1}(r, \varphi, z) \sigma_{a s, i}\right] \\
&+\int_{0}^{2 \pi} \int_{0}^{a} r d r d \phi 2 \sigma_{e s, i} h v_{s} \Delta v N_{2}(r, \varphi) \Gamma_{s, i}(r, \varphi)
\end{aligned}
$$

where $\sigma_{a s, i}$ and $\sigma_{e s, i}$ are the absorption and emission cross-section areas at the $i$-th signal mode, $\Delta v$ is the equivalent amplifying bandwidth, and the $d_{s, i \leftrightarrow k}$ 's are coupling coefficients between signal modes [13]. In the signal propagation Eq. (1), the first term on the right hand side denote net amplification due to stimulated emission, and the second term denote power coupling between the signal modes. In the ASE propagation Eq. (2), the second term on the right hand side represents spontaneous emission of the excited Erbium ions; the coefficient of ' 2 ' preceding this term corresponds to two degenerate polarizations modes. The intensity evolution equation for the power in the $j$-th pump mode at wavelength $\lambda_{p}$ is:

$$
\frac{d P_{p, j}}{d z}=-P_{p, j} \int_{0}^{2 \pi} \int_{0}^{a} r d r d \varphi \Gamma_{p, j}(r, \varphi) N_{1}(r, \varphi, z) \sigma_{a p, j}-\sum_{k=1}^{m_{p}} d_{p, j \leftrightarrow k}\left[P_{p, j}-P_{p, k}\right]
$$

Finally, the population density equations are:

$$
\begin{aligned}
N_{1}(r, \varphi, z)= & \frac{1}{\tau}+\sum_{i=1}^{m_{s}} \frac{\left[P_{s, i}+P_{A S E, i}\right] \sigma_{e s, i} \Gamma_{s, i}(r, \varphi)}{h v_{s}} \\
\frac{1}{\tau}+\sum_{i=1}^{m_{s}} \frac{\left[P_{s, i}+P_{A S E, i}\right]\left(\sigma_{e s, i}+\sigma_{a s, i}\right) \Gamma_{s, i}(r, \varphi)}{h v_{s}}+\sum_{j=1}^{m_{p}} \frac{P_{p, j} \sigma_{a p, j} \Gamma_{p, j}(r, \varphi)}{h v_{p}} & N_{0}(r, \varphi) \\
N_{2}(r, \varphi, z)= & \frac{\sum_{i=1}^{m_{s}} \frac{\left[P_{s, i}+P_{A S E, i}\right] \sigma_{a s, i} \Gamma_{s, i}(r, \varphi)}{h v_{s}}+\sum_{j=1}^{m_{p}} \frac{P_{p, j} \sigma_{a p, j} \Gamma_{p, j}(r, \varphi)}{h v_{p}}}{\frac{1}{\tau}+\sum_{i=1}^{m_{s}} \frac{\left[P_{s, i}+P_{A S E, i}\right]\left(\sigma_{e s, i}+\sigma_{a s, i}\right) \Gamma_{s, i}(r, \varphi)}{h v_{s}}+\sum_{j=1}^{m_{p}} \frac{P_{p, j} \sigma_{a p, j} \Gamma_{p, j}(r, \varphi)}{h v_{p}}} N_{0}(r, \varphi)
\end{aligned}
$$

where $v_{s}$ and $v_{p}$ are the signal and pump optical frequencies. Other symbols are listed in Table 3. Equations (1)-(5) can be solved by using the standard fourth-order Runge-Kutta method given initial conditions for pump and signal power [14]. Gains and noise figures for all signal modes may similarly be calculated.

\section{Simulation}

We consider a MM-EDFA where the EDF has step-index refractive profile. The parameters of the MM-EDFA are shown in Table 1. We assume the doped region is the same size as the core (i.e., $a=r_{c}$ ). As the normalized frequency at $\lambda_{s}=1.53 \mu \mathrm{m}$ lies between $2.405<V_{s}<3.832$, the EDF supports two degenerate mode groups at this wavelength. We assume a weakly guiding MMF where the modes are well approximated by linearly polarized (LP) modes [15].

\#147622 - \$15.00 USD Received 16 May 2011; revised 27 Jun 2011; accepted 7 Jul 2011; published 15 Aug 2011 (C) 2011 OSA 
For the remainder of this paper, we use the notation $\mathrm{LP}_{i j, s}$ and $\mathrm{LP}_{x y, p}$ to denote the $\mathrm{LP}_{i j}$ mode at $\lambda_{s}$ and $\mathrm{LP}_{x y}$ mode at $\lambda_{p}$, respectively. Figure 3 shows the intensity profiles of the modes, and their intensities viewed along the $x$-axis. We note that for $m>0$, the $\mathrm{LP}_{\mathrm{mn}, \mathrm{s}}$ and $\mathrm{LP}_{\mathrm{mn}, \mathrm{p}}$ have two spatially degenerate modes. In one of these modes, referred to as the "even" mode, intensity is maximized along the $\mathrm{x}$-axis at $(\varphi=0)$; in the other mode, referred to as the "odd mode", intensity is minimized along the $\mathrm{x}$-axis. All spatial modes, degenerate and nondegenerate, come with two degenerate polarization modes.

Table 1. Parameters of a MM-EDFA

\begin{tabular}{llll}
\hline Parameter & \multicolumn{1}{c}{ Value } & Parameter & Value \\
\hline$r_{c}(\mu m)$ & 8 & $\sigma_{a s, i}\left(m^{2}\right)$ & $5.64 \times 10^{-25}$ \\
$N A$ & 0.1 & $\sigma_{e s, i}\left(m^{2}\right)$ & $5.71 \times 10^{-25}$ \\
$N_{0}\left(m^{-3}\right)$ & $1 \times 10^{24}$ & $\sigma_{a p, i}\left(m^{2}\right)$ & $2.86 \times 10^{-25}$ \\
$\tau(m s)$ & 10 & $\sigma_{e p, i}\left(m^{2}\right)$ & 0 \\
$\lambda_{s}(\mu m)$ & 1.53 & $\lambda_{p}(\mu m)$ & 0.98 \\
$L(m)$ & 30 & & \\
\hline
\end{tabular}

\subsection{Modal Gain Control for Non-Degenerate Signal Modes}

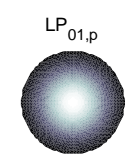

$\mathrm{LP}_{21 \mathrm{e}, \mathrm{p}}$

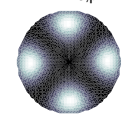

$y \quad \mathrm{LP}_{01, \mathrm{~s}}$

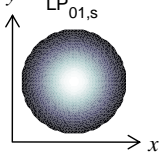

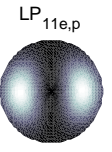

$\mathrm{LP}_{210, \mathrm{p}}$
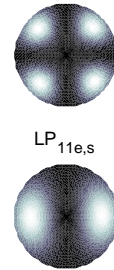
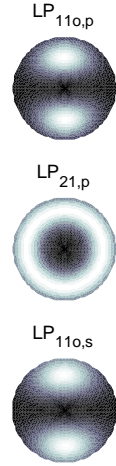

(a)

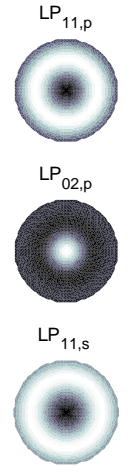

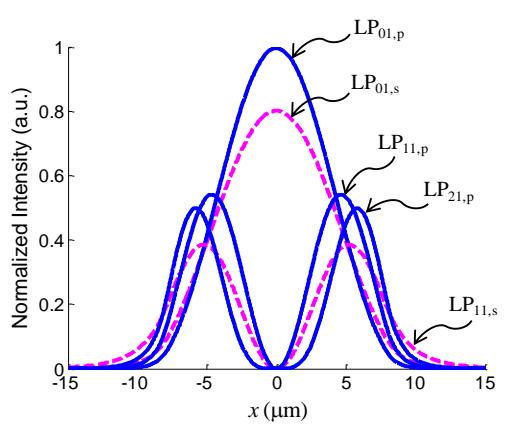

(b)

Fig. 3. (a) Intensity profile of pump and signal modes, (b) normalized intensity profiles viewed along $x$-axis.

We first consider MDG using a single-mode pump. For the spatially degenerate $\mathrm{LP}_{11, p}$ and $\mathrm{LP}_{21, p}$ modes, we assume equal power in the even $\left(\mathrm{LP}_{11 e, p}\right.$ or $\left.\mathrm{LP}_{21 e, p}\right)$ and odd modes $\left(\mathrm{LP}_{11 \rho, p}\right.$ or $\mathrm{LP}_{21 o, p}$ ) so that the resulting intensity (power) patterns (e.g., $\mathrm{LP}_{11, p}=\frac{1}{2}\left(\mathrm{LP}_{11 e, p}+\mathrm{LP}_{110, p}\right)$ ) have no azimuthal dependence (Fig. 3(a)), and hence no MDG between spatially degenerate signal modes. Figure 4 shows the gain experienced by each signal mode group when pumping in the $\mathrm{LP}_{01, \mathrm{p}}, \mathrm{LP}_{11, \mathrm{p}}$ and $\mathrm{LP}_{21, \mathrm{p}}$ modes. It is assumed that the input signal to the EDF has equal power $\left(0.05 \mathrm{~mW}\right.$ ) in each of its six (two $\mathrm{LP}_{01, \mathrm{~s}}$ and four $\mathrm{LP}_{11, \mathrm{~s}}$ ) spatial and polarization degenerate modes, or $0.3 \mathrm{~mW}$ in total. Since the intensity profile of $\mathrm{LP}_{01, \mathrm{p}}$ is better matched to $\mathrm{LP}_{01, \mathrm{~s}}$ than $\mathrm{LP}_{11, \mathrm{~s}}$, Fig. 4(a) shows higher gain for $\mathrm{LP}_{01, \mathrm{~s}}$. Conversely, pumping in $\mathrm{LP}_{21, \mathrm{p}}$ results in higher gain for $\mathrm{LP}_{11, \mathrm{~s}}$. 

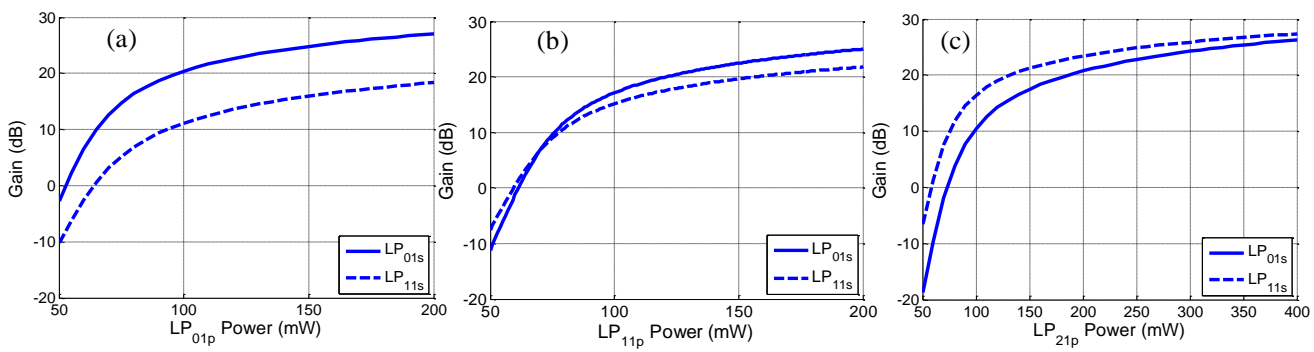

Fig. 4. Modal gain of signal at $1530 \mathrm{~nm}$ assuming $0.05 \mathrm{~mW}$ power in each degenerate modes of $\mathrm{LP}_{01, s}$ and $\mathrm{LP}_{11, s}$, when 980-nm pump is entirely confined in (a) $\mathrm{LP}_{01, p}$, (b) $\mathrm{LP}_{11, p}$ and (c) $\mathrm{LP}_{21, p}$.

The quality of the match between signal and pump intensity profiles can be evaluated by the overlap integral:

$$
\eta_{p j, s i}=\int_{0}^{2 \pi} \int_{0}^{a} r d r d \varphi \Gamma_{p, j}(r, \varphi) \Gamma_{s, i}(r, \varphi)
$$

Table 2 shows $\eta_{p j, s i}$ for different pump and signal mode pairs.

Table 2. Overlap Integrals of Normalized Intensity Profile

\begin{tabular}{ccc}
\hline (m-2) & $\mathrm{LP}_{01, s}$ & $\mathrm{LP}_{11, s}$ \\
\hline $\mathrm{LP}_{01, p}$ & $6.2449 \times 10^{9}$ & $3.2848 \times 10^{9}$ \\
$\mathrm{LP}_{11, p}$ & $4.4242 \times 10^{9}$ & $3.7472 \times 10^{9}$ \\
$\mathrm{LP}_{21, p}$ & $3.2498 \times 10^{9}$ & $3.4857 \times 10^{9}$ \\
\hline
\end{tabular}

It is observed that $\eta_{p 01, s 01}$ and $\eta_{p 11, s 01}$ are both larger than $\eta_{p 01, s 11}$ and $\eta_{p 11, s 11}$. Hence, higher gain is observed for $\mathrm{LP}_{01, s}$ when these pump modes are used. Conversely, as $\eta_{p 21, s 11}$ is larger than $\eta_{p 21, s 01}$, pumping in $\mathrm{LP}_{21, p}$ gives higher gain for $\mathrm{LP}_{11, s}$. It is possible to control MDG by varying the relative powers of $\mathrm{LP}_{01, p}$ and $\mathrm{LP}_{21, p}$. In transmission, the higher-order $\mathrm{LP}_{11, s}$ mode is less confined by the core, and will experience higher bending loss than the fundamental $\mathrm{LP}_{01, s}$ mode. Furthermore, the $\mathrm{LP}_{11, s}$ mode has larger effective area, making the optimum power for this mode higher than the fundamental mode. Consequently, a practical MM-EDFA will pump primarily in the $\mathrm{LP}_{21, p}$. The addition of a small amount of $\mathrm{LP}_{01, p}$ enables adjustment of MDG. Figure 5(a) shows modal gain versus $\mathrm{LP}_{21, p}$ pump power, where the power of $\mathrm{LP}_{01, p}$ was continually adjusted to maintain a $1 \mathrm{~dB}$ difference between the gains of $\mathrm{LP}_{01, s}$ and $\mathrm{LP}_{11, s}$, which we denoted as $\Delta \mathrm{G}_{11 s-01 s}$. Figure 5(b) shows the same results for $\Delta \mathrm{G}_{11 s-01 s}=2 \mathrm{~dB}$. It is observed that modal gain can be continually adjusted to values greater than $22 \mathrm{~dB}$, which is sufficient to compensate the loss of a single fiber span at typical span distances. Figure 5(c) shows the sensitivity of modal gain to $\mathrm{LP}_{01, p}$ power when $\mathrm{LP}_{21, p}$ power is fixed at $150 \mathrm{~mW}$. While the modal gain at $\mathrm{LP}_{11, s}$ remain nearly constant, $\Delta \mathrm{G}_{11 s-01 s}$ varies by more than $4 \mathrm{~dB}$ as $\mathrm{LP}_{01, p}$ power is changed from only 0 to $20 \mathrm{~mW}$, demonstrating wide tunability of MDG in dynamic range. Thus, to establish the desired modal gain in an MM-EDFA, we first tune the power of the $\mathrm{LP}_{21, p}$ pump to give the desired power for the $\mathrm{LP}_{11, s}$ mode, after which, the power of $\mathrm{LP}_{01, p}$ is adjusted to obtain the desired $\Delta \mathrm{G}_{11 s-01 s}$. 

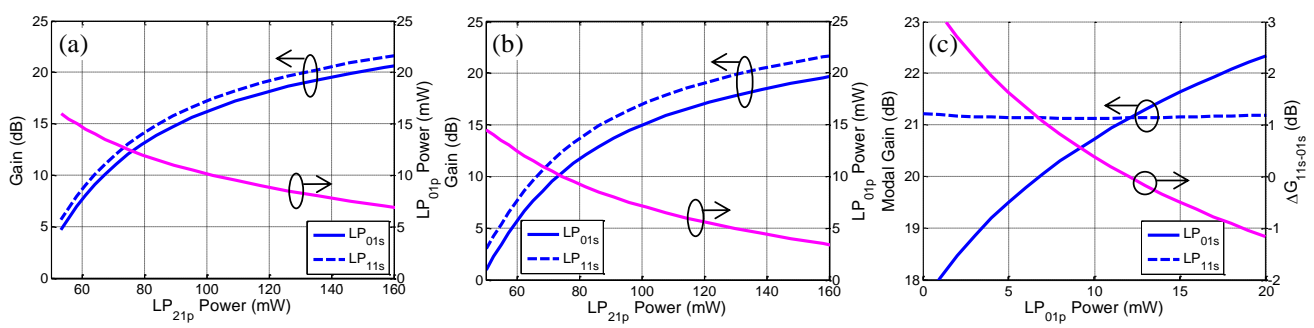

Fig. 5. Modal gain and required $\mathrm{LP}_{01, p}$ power vs. $\mathrm{LP}_{21, p}$ power, to maintain MDG $\left(\Delta \mathrm{G}_{11 s-01 s}\right)$ at (a) $1 \mathrm{~dB}$ and (b) $2 \mathrm{~dB}$; (c) Modal gain and MDG vs. LP ${ }_{01, p}$ power for fixed $\mathrm{LP}_{21, p}$ power at 150 $\mathrm{mW}$.

MDG depends not only on pump power, but also the length of the EDF. Figure 6 shows modal gain vs. EDF length for two pump configurations. At short fiber lengths, modal gain increases with increasing EDF length. Eventually, pump depletion and high signal power depletes population inversion, so modal gain decreases for further increases in EDF length. For any given EDF length, $\Delta \mathrm{G}_{11 s-01 s}$ is maximized when only the $\mathrm{LP}_{21, p}$ mode is pumped. Figure 6(a) shows that a longer EDF gives larger $\Delta \mathrm{G}_{11 s-01 s}$, and thus larger range of achievable MDG. We can select an EDF length which approximately maximizes modal gain, while ensuring relatively large difference between the gains of $\mathrm{LP}_{11, s}$ and $\mathrm{LP}_{01, s}$. In Fig. 6(b), it is observed that adding only $8 \mathrm{~mW}$ of pump power in $\mathrm{LP}_{01, p}$ enables flattening of the modal gains responses. Even as device length is swept from 20 to 40 meters, less than $\pm 1 \mathrm{~dB}$ change in the modal gains of $\mathrm{LP}_{11, s}$ and $\mathrm{LP}_{01, s}$, and less than $\pm 0.5 \mathrm{~dB}$ variation in $\mathrm{MDG}$ are observed.
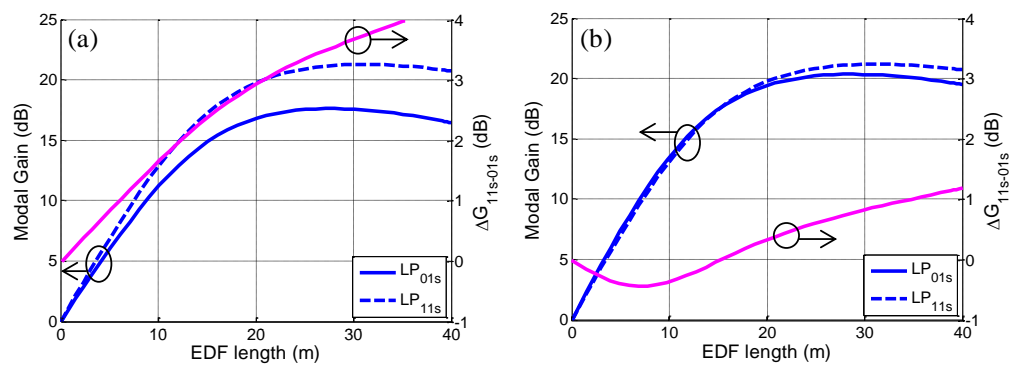

Fig. 6. Modal gain and MDG difference vs. EDF length, when $\mathrm{LP}_{01, p}$ and $\mathrm{LP}_{21, p}$ have powers of: (a) $P_{p, 21}=150 \mathrm{~mW}, P_{p, 01}=0 \mathrm{~mW}$, and (b) $P_{p, 21}=150 \mathrm{~mW}, P_{p, 01}=8 \mathrm{~mW}$.

\subsection{Modal Gain Control for Spatially Degenerate Signal Modes}

In 3.1 section, it was assumed that odd and even modes of the spatially degenerate $\mathrm{LP}_{11, p}$ and $\mathrm{LP}_{21, p}$ modes are pumped with equal power, resulting in no MDG between spatially degenerate signal modes such as $\mathrm{LP}_{11, s .}$. In practical MDM systems, however, spatially degenerate signal modes may have different losses. One mechanism for MDL is fiber bending, where the loss experienced by each spatially degenerate mode depends on its orientation relative to the plane containing the bend [16]. For our MM-EDFA to overcome $\mathrm{MDL}$, the gains for $\mathrm{LP}_{11 \mathrm{e}, s}$ and $\mathrm{LP}_{11 o, s}$ need to be adjustable. It was previously observed in Fig. 4 that modal gain is related to the intensity overlap between the pump mode and the signal mode in question. The assumption of equal pump power in even \& odd modes for $\mathrm{LP}_{11, p}$ or $\mathrm{LP}_{21, p}$ caused the overlap integral to be independent of azimuthal angle. We now consider a pump with azimuthal intensity dependence. Consider a rotated $\mathrm{LP}_{11 e, p}$ pump as shown in Fig. 7, which we denote as $\mathrm{LP}_{11 \theta, p}$. It can be shown that the overlap intensity integral between a rotated pump mode $\mathrm{LP}_{m k \theta, p}$ (with $m$ azimuthal nulls) and an even signal mode $\mathrm{LP}_{n j e, s}$ with $n$ azimuthal nulls is proportional to:

\#147622 - \$15.00 USD Received 16 May 2011; revised 27 Jun 2011; accepted 7 Jul 2011; published 15 Aug 2011

(C) 2011 OSA

15 August 2011/Vol. 19, No. 17 / OPTICS EXPRESS 16607 


$$
\eta_{p(m k), s(n j)} \propto \int_{0}^{2 \pi} d \varphi \cos ^{2}(m \varphi) \cos ^{2}(n(\varphi+\theta))=\frac{\pi}{2}+\cos (2 n \theta) \delta(m-n)
$$

where $\varphi$ is the azimuthal angle, $\delta(\cdot)$ is the Kronecker delta function, and $\theta$ is the angular offset. It is observed that if pump and signal has different azimuthal mode number (i.e., $m \neq n$ ), the overlap integral is independent of $\theta$. Otherwise, the overlap integral - and hence MDG will have $\theta$ dependence. Figure 8(a) shows the $\theta$ dependence of modal gain assuming we pump only in the $\mathrm{LP}_{11 \theta, \mathrm{p}}$ mode. As the rotation angle $\theta$ is scanned from 0 to $2 \pi$, the modal gains for $\mathrm{LP}_{11 e, s}$ and $\mathrm{LP}_{11 o, s}$ oscillates with a period of $\pi$, reflecting the rotational symmetry of the $\mathrm{LP}_{11 \theta, p}$ mode. When the intensity peaks of the $\mathrm{LP}_{11 \theta, p}$ pump align with $\mathrm{LP}_{11 e, s}$, its gain is maximized while the gain of $\mathrm{LP}_{11 o, s}$ is minimized, and vice-versa. We observed that MDG between these modes can be as much as $20 \mathrm{~dB}$. Meanwhile, the fluctuation in gain for the $\mathrm{LP}_{01, s}$ mode is a second order effect caused by gain competition between the signal modes; when the pump is at $45^{\circ}$ relative to both the $\mathrm{LP}_{11 e, s}$ and $\mathrm{LP}_{110, s}$ modes, maximum gain occurs for $\mathrm{LP}_{01, s}$. In Fig. 8(b), we assume pumping in only $\mathrm{LP}_{21 \theta, \mathrm{p}}$. In this pumping regime, the rotation angle $\theta$ has no impact on MDG, as expected from the overlap integral in Eq. (7), which is constant when $m \neq n$. Finally, Fig. 8(c) shows MDG for a pump regime similar to one in a real implementation, where the pump is mostly in $\mathrm{LP}_{21, \mathrm{p}}$ (as defined in Fig. 3(a)), with small amounts of $\mathrm{LP}_{01}, \mathrm{p}$ and $\mathrm{LP}_{11 \mathrm{e}, \mathrm{p}}$ added to adjust the MDG between the three modes. The results show that the gain difference between $\mathrm{LP}_{11 \mathrm{e}, \mathrm{s}}$ and $\mathrm{LP}_{11 \mathrm{o}, \mathrm{s}}$ can be tuned by proper rotation of the $\mathrm{LP}_{11 \theta, \mathrm{p}}$, while the variation in gain for $\mathrm{LP}_{01, \mathrm{~s}}$ is as small as $\pm 0.05 \mathrm{~dB}$.
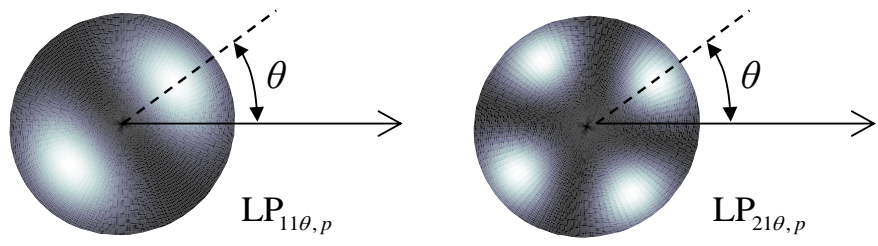

Fig. 7. Rotated pump modes.
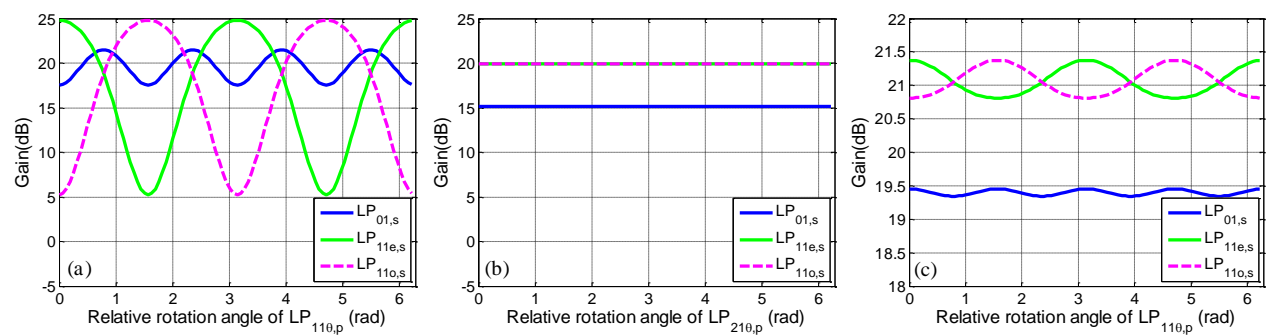

Fig. 8. Modal gain vs. relative rotation angle $(\theta)$ of the pump mode when pump powers are: (a)

$P_{p, 11 \theta}=150 \mathrm{~mW}$, (b) $P_{p, 21 \theta}=150 \mathrm{~mW}$, and (c) $P_{p, 21}=150 \mathrm{~mW}, P_{p, 01}=2 \mathrm{~mW}, P_{p, 11 \theta}=15 \mathrm{~mW}$

\subsection{Impact on Performance Due to Inexact Excitation and Mode Coupling}

Previously, it was assumed that (a) the pump can be excited in particular modes of the EDF without leakage into undesired mode, and (b) no mode-coupling between different pump modes during propagation inside the EDF. In practice, either of these assumptions may be difficult to satisfy. Firstly, to generate the desired pump mode (Fig. 1), phase plates are likely to be used - as was demonstrated in an MDM transmission experiment in [9]. A phase plate alone (without modulation of transverse amplitude) will result in excitation of other unwanted pump modes. Additionally, spatial misalignment between pump and signal at the beam combiner (shown as the dichroic mirror in Fig. 1) will cause further energy leakage into 
unwanted modes and excess loss, leading to performance degradation. We consider only leakage from $\mathrm{LP}_{21, p}$ to the other modes, as $\mathrm{LP}_{21, p}$ has the most power. Figure 9 shows the modal gains in $\mathrm{LP}_{01, s}$ and $\mathrm{LP}_{11, s}$ versus the percentage of $\mathrm{LP}_{21, p}$ power leaked into the unwanted modes. Note the power in $\mathrm{LP}_{01, p}$ is chosen to give an $\mathrm{MDG}$ of $\Delta \mathrm{G}_{11 s-01 s}=2 \mathrm{~dB}$ when $0 \%$ of $\mathrm{LP}_{21, p}$ is coupled to unwanted modes. As previously shown in Fig. 4, pumping in the other modes gives higher gain for $\mathrm{LP}_{01, s}$ than $\mathrm{LP}_{11, s}$. Hence we observe a reduction in $\Delta \mathrm{G}_{11 s-01 s}$ that as leakage increases. At sufficiently high leakage, $\Delta \mathrm{G}_{11 s-01 s}$ become negative, indicating higher modal gain for $\mathrm{LP}_{01, s}$. It is also observed that $\Delta \mathrm{G}_{11 s-01 s}$ has greater sensitivity to leakage into $\mathrm{LP}_{01, p}$ and $\mathrm{LP}_{02, p}$, as these modes have larger overlap integrals with $\mathrm{LP}_{01, s}$ than $\mathrm{LP}_{11, p}$ (Table 2). Hence, the pump generation mechanism should minimize leakage into these modes.
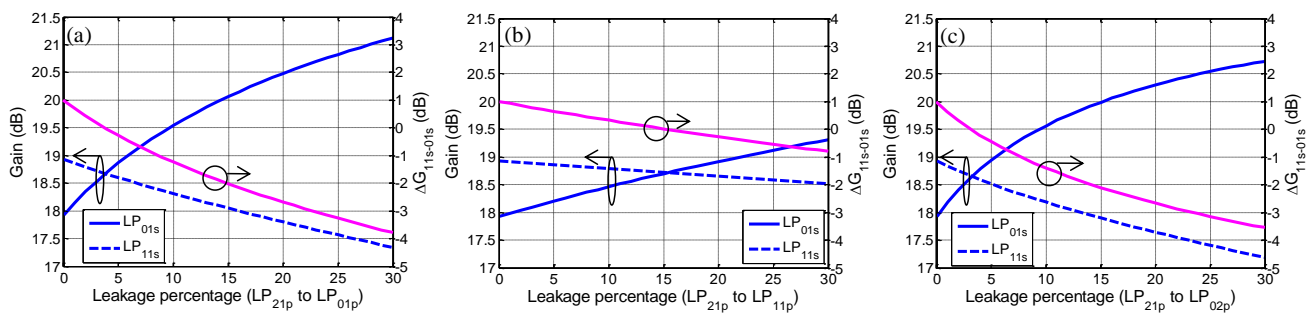

Fig. 9. Modal gain vs. power leakage, (a) $\mathrm{LP}_{21, p}$ to $\mathrm{LP}_{01, p}$, (b) $\mathrm{LP}_{21, p}$ to $\mathrm{LP}_{11, p}$, (c) $\mathrm{LP}_{21, p}$ to $\mathrm{LP}_{02, p}$.



Fig. 10. Modal gain vs. mode coupling strength from $\operatorname{LP}_{21, p}$ to $\operatorname{LP}_{01, p}\left(d_{p, 21 \leftrightarrow 02}\right)$.

Mode coupling during propagation inside the EDF will have similar impact as excitation of unwanted modes, since both result in power transferred into undesired modes. The strength of mode coupling is inversely proportional to the difference in effective refractive index between the modes $\left(\Delta n_{\text {eff }}\right)$. Hence, we focus on coupling between $\mathrm{LP}_{21, p}$ and $\mathrm{LP}_{02, p}$, as the effective refractive index difference $\Delta n_{\text {eff,(2lp-02p) }}$ between this pair of modes is the smallest among all the mode pairs in our FMF-based EDF. Assuming the power coupling coefficient between $\mathrm{LP}_{21, p}$ and $\mathrm{LP}_{02, p}$, which was defined as $d_{p, 21 \leftrightarrow 02}$ in Eq. (3), is constant throughout the EDF, Fig. 10 shows MDG as a function of $d_{p, 21 \leftrightarrow 02}$. The value of coupling coefficient refers to [13]. As expected, $\Delta \mathrm{G}_{11 \mathrm{~s}-01 \mathrm{~s}}$ decreases with increasing strength of mode coupling. This favors using a shorter length EDF, and a refractive index profile that maximizes effective refractive index difference between the modes to reduce mode coupling.

\subsection{Impact on Performance Due to Macro-Bending Loss}

In the results thus far, the loss of EDF is assumed to be negligible. In practice, however, the EDF has to be spooled to create a module, which may introduce macro-bending loss. As the higher order $\mathrm{LP}_{11, s}$ and $\mathrm{LP}_{21, p}$ modes are less confined, they are more likely to couple into cladding modes when the fiber is bent, resulting in higher macro-bending loss than the fundamental modes $\mathrm{LP}_{01, s}$ and $\mathrm{LP}_{01, p}$. This must either be taken into account by increasing the power of the higher order pump, or the bending radius has to be large enough to render 
bending loss negligible. The theoretical macro-bending loss can be calculated using Marcuse's curvature loss formula [17]. Figure 11 shows the macro-bending losses for the various signal and pump modes as functions of bend radius.

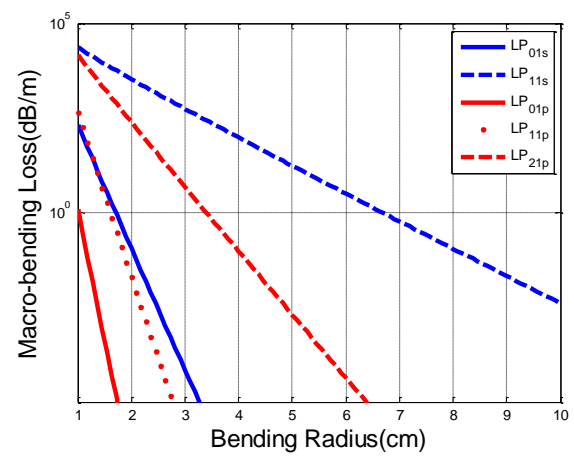

Fig. 11. Macro-bending loss vs. bending radius.

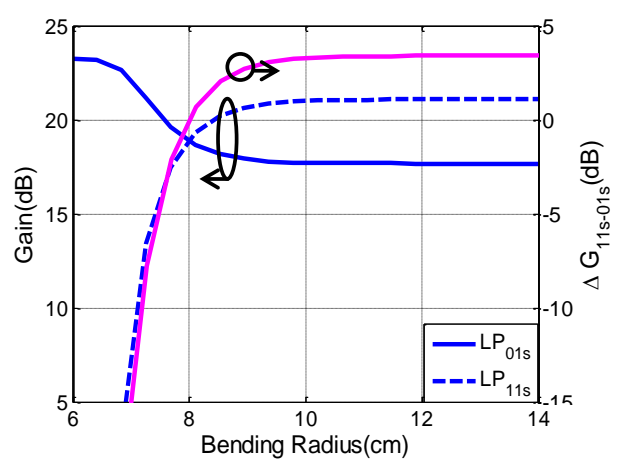

Fig. 12. Modal gain vs. mode dependent loss of $\mathrm{LP}_{11 \mathrm{~s}}$.

It is observed that macro-bending loss of all modes grows exponentially as bending radius decreases. In particular, the $\mathrm{LP}_{11, s}$ mode is the least spatially confined. To reduce its macrobending loss to less than $0.01 \mathrm{~dB} / \mathrm{m}$, the bending radius must be at least $9.46 \mathrm{~cm}$. Figure 12 shows modal gain as a function of bend radius assuming only $\mathrm{LP}_{21 p}$ is pumped at a power of $150 \mathrm{~mW}$. It is observed that the gain of $\mathrm{LP}_{11, s}$ is reduced significantly as bend radius falls below $9 \mathrm{~cm}$. In particular, MDG between the modes is reduced to zero at a bend radius of 8 $\mathrm{cm}$, making it impossible to equalize MDL in the transmission by pumping in $\mathrm{LP}_{01 p}$ as outlined in Section 3.1. It is observed that at low bend radius, the gain of $\mathrm{LP}_{01, s}$ is increased due to reduced mode competition. However, the increase in gain saturates when $\mathrm{LP}_{11, s}$ is completely stripped out. At very small bend radius, macro-bending loss will again reduce the gain of $\mathrm{LP}_{01, s}$. The results indicate that a practical EDF should be spooled with a bend radius greater than $9 \mathrm{~cm}$ for a step-index fiber design. If device size is an issue, it is also possible to create fibers more complex refractive index profiles, such as using refractive index trenches, to better confine all signal and pump modes.

\section{Conclusion}

A multimode EDFA with modal gain control is proposed. By adjusting relative amount of $\mathrm{LP}_{01, p}$ and $\mathrm{LP}_{21, p}$, the gains of the $\mathrm{LP}_{01, s}$ and $\mathrm{LP}_{11, s}$ signal modes can be tuned over a wide dynamic range. The relative gain between the two spatially degenerate $\mathrm{LP}_{11, s}$ signal modes can also be adjusted by adding a small amount of $\mathrm{LP}_{11 \theta, p}$ which is the even $\mathrm{LP}_{11 e, p}$ mode rotated by angle $\theta$. Performance impact due to excitation of unwanted pump modes at the input of the 
EDF, mode coupling and macro-bending loss in the fiber was also investigated. The proposed modal gain control scheme can be generalized for an $N$-mode MM-EDFA by varying the powers of $N$ well-chosen pump modes.

\section{Appendix}

Table 3. List of Variables Used in the Coupled Eqs. (1)-(5)

\begin{tabular}{|c|c|c|c|}
\hline Symbol & Definition & Symbol & Definition \\
\hline$\Gamma_{s, i}(r, \varphi)$ & $\begin{array}{l}\text { Normalized intensity } \\
\text { profile for } i \text {-th mode at } \\
\lambda_{s}\end{array}$ & $\Gamma_{p, j}(r, \varphi)$ & $\begin{array}{l}\text { Normalized intensity } \\
\text { profile for } j \text {-th mode at } \\
\lambda_{p}\end{array}$ \\
\hline$N_{1}(r, \varphi, z)$ & $\begin{array}{l}\text { Erbium ion population } \\
\text { density of lower level }\end{array}$ & $N_{2}(r, \varphi, z)$ & $\begin{array}{l}\text { Erbium ion population } \\
\text { density of upper level }\end{array}$ \\
\hline$N_{0}(r, \varphi)$ & $\begin{array}{l}\text { Erbium ion doping } \\
\text { density }\end{array}$ & $h$ & Planck constant \\
\hline$\sigma_{a s, i}$ & $\begin{array}{l}\text { Absorption cross- } \\
\text { section for } i \text {-th mode at } \\
\lambda_{s}\end{array}$ & $\sigma_{a p, j}$ & $\begin{array}{l}\text { Absorption cross- } \\
\text { section for } j \text {-th mode at } \\
\lambda_{p}\end{array}$ \\
\hline$\sigma_{e s, i}$ & $\begin{array}{l}\text { Emission cross-section } \\
\text { for } i \text {-th mode at } \lambda_{s}\end{array}$ & $\tau$ & $\begin{array}{l}\text { Spontaneous emission } \\
\text { lifetime for the excited } \\
\text { state }\end{array}$ \\
\hline$m_{s}$ & $\begin{array}{l}\text { Total number of guided } \\
\text { modes at } \lambda_{s}\end{array}$ & $m_{p}$ & $\begin{array}{l}\text { Total number of guided } \\
\text { modes at } \lambda_{p}\end{array}$ \\
\hline$v_{s}$ & $\begin{array}{l}\text { Optical frequency of } \\
\text { the signal at } \lambda_{s}\end{array}$ & $v_{p}$ & $\begin{array}{l}\text { Optical frequency of } \\
\text { the pump at } \lambda_{p}\end{array}$ \\
\hline$\Delta v$ & $\begin{array}{l}\text { Equivalent amplifying } \\
\text { bandwidth }\end{array}$ & $P_{s, i}$ & $\begin{array}{l}\text { Power of the signal for } \\
i \text {-th mode at } \lambda_{s}\end{array}$ \\
\hline$P_{p, j}$ & $\begin{array}{l}\text { Power of the pump for } \\
j \text {-th mode at } \lambda_{p}\end{array}$ & $P_{A S E, i}$ & $\begin{array}{l}\text { Power of ASE for } i \text {-th } \\
\text { mode }\end{array}$ \\
\hline$d_{s, i \leftrightarrow k}$ & $\begin{array}{l}\text { Mode coupling } \\
\text { coefficient between } i \text {-th } \\
\text { and } k \text {-th mode of the } \\
\text { signal at } \lambda_{s}\end{array}$ & $d_{p, j \leftrightarrow k}$ & $\begin{array}{l}\text { Mode coupling } \\
\text { coefficient between } j \text {-th } \\
\text { and } k \text {-th mode of the } \\
\text { pump at } \lambda_{p}\end{array}$ \\
\hline$r_{c}$ & $\begin{array}{l}\text { Core radius of Erbium- } \\
\text { doped fiber }\end{array}$ & $a$ & $\begin{array}{l}\text { Radius of uniform } \\
\text { doping region }\end{array}$ \\
\hline$\Delta \mathrm{G}_{11 s-01 s}$ & $\begin{array}{l}\text { Mode-dependent gain } \\
\text { (MDG): Gain of } \mathrm{LP}_{11, s} \\
\text { minus gain of } \mathrm{LP}_{01, s}\end{array}$ & & \\
\hline
\end{tabular}

\#147622 - \$15.00 USD Received 16 May 2011; revised 27 Jun 2011; accepted 7 Jul 2011; published 15 Aug 2011 